immune complexes (IC), and autoantibodies are supposed to participate in IC formation. Thus, the fraction of autoantibodies participating in IC formation merits interest as a diagnostic and/or prognostic marker. We have developed a technique to quantify autoantibody content in IC (Sohrabian et al. Ann Rheum Dis 2015;74(Suppl 1):A74).

Objectives: To evaluate quantification of autoantibodies in IC as a measure of disease activity and prognosis for response in belimumab-treated SLE patients. Methods: Fifty-five SLE cases classified according to the ACR criteria were treated with belimumab and followed for one year. High disease activity was defined as SLE Disease Activity Index 2000 (SLEDAI-2K) $\geq 10$, or as low levels of complement factors C3 and/or C4. Treatment responses were recorded using the SLE Responder Index (SRI), or as a value 0 for modified SLEDAI-2K with suppression of autoantibody and complement data (clinical remission). Low disease activity was also recorded as Lupus Low Disease Activity State (LLDAS). Response data were recorded at 3,6 and 12 months.

IC were purified from sera by binding to $\mathrm{C} 1 \mathrm{q}$-coated beads, and thereafter eluted. Autoantibody levels were determined in unmodified serum and in solubilised IC with addressable laser bead immunoassay (FIDIS Connective, Theradiag, Paris) for autoantibodies against dsDNA, histones, ribosomal $P$ antigen, proliferating cell nuclear antigen (PCNA), SSA/Ro60, SSA/Ro52, SSB, Sm, U1RNP and the $\mathrm{Sm} / \mathrm{RNP}$ complex. Autoantibody levels in serum and in IC were compared with Mann-Whitney's $U$ test between patients with high and low disease activity at baseline and between patients with and without treatment response during the follow-up period.

Results: Antibodies against dsDNA, SSA/Ro60 and Sm/RNP were found in 65\%, $54 \%$ and $43 \%$, other antibodies with lower percentages. Low complement levels were associated with high serum anti-dsDNA ( $\mathrm{p}=0.003)$ and anti-ribosomal $\mathrm{P}$ antigen $(p=0.008)$ levels, whereas high SLEDAI-2K associated with high anti-dsDNA $(p=0.02)$ and anti-Sm/RNP $(p=0.047)$ levels. Serum levels of antibodies against SSA/Ro60, SSB and SSA/Ro52 were lower in patients attaining LLDAS after 6 months ( $p=0.02,0.051$ [trend] and 0.04 , respectively); these associations were stronger for corresponding IC fractions ( $\mathrm{p}=0.01,0.005$, and 0.002 , respectively). Baseline levels of anti-dsDNA and anti-histones in IC associated with clinical remission ever during the follow-up period $(p=0.003$ and $p=0.05)$. Low levels of anti-Sm and anti-Sm/RNP in serum but not in IC associated with clinical remission at month $6(p=0.02$ and 0.04 respectively), and for Sm/RNP also at month 3 $(\mathrm{p}=0.04)$.

Serum levels of all antibodies except SSA/Ro52 declined during the first 6 months, most prominently for dsDNA, histones ribosomal P, PCNA and Sm/RNP $(p<0.0001$ for all). Levels of antibodies in IC declined only for dsDNA $(p=0.048)$.
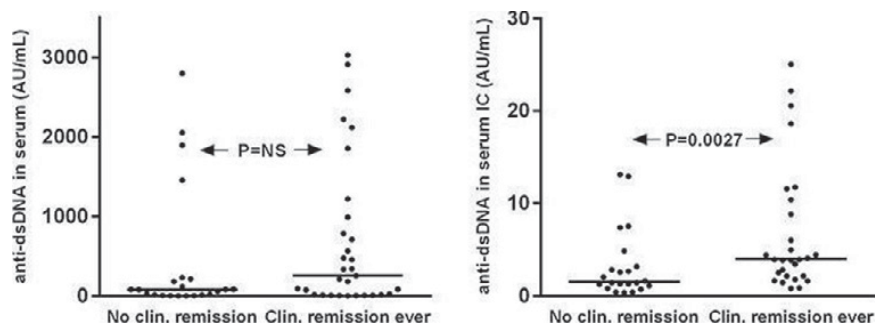

Conclusions: Autoantibody levels in serum and in IC show different associations to disease activity and to treatment response. High baseline anti-dsDNA levels in IC were most strongly associated with clinical remission, and decreased during belimumab treatment. Belimumab effect might primarily relate to autoantibodies in IC.

Disclosure of Interest: None declared

DOI: 10.1136/annrheumdis-2017-eular.5054

\section{SAT0234 PROSPECTIVE SINGLE CENTRE STUDY OF EFFECTIVENESS OF UPFRONT RITUXIMAB AND MYCOPHENOLATE WITH MINIMUM STEROID IN SLE}

B.G. Dharmanand. Rheumatology, Sakra World Hospital, Sy No.52/2 \& 52/3, Devarabeesanahalli, Oppsite Intel, Varthur Hobli, Bengaluru, Karnataka 560103, Bangalore, India

Background: Treatment options for SLE have significant morbidity and mortality. Side effects from corticosteroid usage limit patient adherence and treatment efficacy. B cell depletion appears to target a critical pathophysiological pathway in SLE. Trails with rituximab have shown mixed results. Rituxilup study demonstrated efficacy and steroid sparing action of rituximab and mycophenolate (1)

Objectives: We aim to analyse our experience of using rituximab and mycophenolate upfront on presentation with minimum oral steroids.

Methods: 12 patients with SLE, seen between Jan 2015 to march 2016, were included in the study. All patients completed 6 months of follow-up. Patients were treated with 2 doses of rituximab $(1 \mathrm{~g})$ and methyl prednisolone $(500 \mathrm{mg})$ on days 1 and 15 , and maintenance treatment of mycophenolate mofetil (2000mg) and low dose prednisolone $(<7.5 \mathrm{mg}$ ) which was tapered off.

Results: 10 were females and 2 males. Mean age of the patients is 24.5 . 9 had lupus nephritis, 1 mesenteric vasculitis, 1 CNS vasculitis and 1 severe cutaneous vasculitis with pancytopenia. Average SLEDAI improved from 14 to 4 . 6/9 LN attained complete renal remission and 2 partial remission. one patient died due to infection and renal disease 15 days after infusion. 2 vasculitis and one NPSLE patient improved completely. Two patients had infection requiring hospitalization with in 8 weeks of infusion and one patient had severe bradycardia during the infusion and received only $1000 \mathrm{mg}$ rituximab. Steroid was stopped by 6 months in 6 patients and in the dose was below $5 \mathrm{mg}$ in rest.

Conclusions: Early Rituximab and mycophenolate is an effective option for treating severe lupus and has steroid sparing property.

\section{References:}

[1] Condon MB, Ashby D, Pepper RJ, et al. Prospective observational singlecentre cohort study to evaluate the effectiveness of treating lupus nephritis with rituximab and mycophenolate mofetil but no oral steroids. Ann Rheum Dis (2013) 72:1280-1286.

Disclosure of Interest: None declared

DOI: 10.1136/annrheumdis-2017-eular.2902

\section{SAT0235 BIIB059, A MONOCLONAL ANTIBODY TARGETING BCDA2 SHOWS SAFETY, TOLERABILITY, PHARMACOKINETICS, PHARMACODYNAMICS IN HEALTHY VOLUNTEER SUBJECTS}

D. Martin ${ }^{1}$, L. Stevenson ${ }^{1}$, P. Prasad ${ }^{1}$, K. Smirnakis ${ }^{1}$, A. Kao ${ }^{1}$, D. Rabah ${ }^{1}$, W. Wang ${ }^{1}$, C. Barbey ${ }^{2}$, C. Musselli ${ }^{1}$, N. Franchimont ${ }^{1} .{ }^{1}$ Biogen, Cambridge, United States; ${ }^{2}$ Biogen, Zug, Switzerland

Background: Type I interferons (IFN-I) are central to the pathogenesis of systemic lupus erythematosus (SLE). BDCA2 is a plasmacytoid dendritic cell (pDC)-specific receptor that, upon engagement, inhibits the production of IFN-I and other inflammatory mediators. Targeting BDCA2 therefore represents an attractive therapeutic strategy for inhibiting PDC-derived IFN-I and may be an effective therapy for the treatment of SLE. BIIB059, an investigational anti-BDCA2 humanized monoclonal antibody, has been shown to engage BDCA2, and this interaction leads to BDCA2 internalization and the consequent in vitro inhibition of TLR-induced IFN-I by pDCs (Pellerin 2015).

Objectives: This study is a first-in-human study that aimed to assess safety, tolerability, PK and PD of BIIB059 in HV subjects following administration of single ascending doses of BIIB059.

Methods: A randomized, double-blinded, placebo controlled, single ascending dose-escalation was conducted in adult HV. Subjects received a single administration of either BIIB059 or placebo. BIIB059 was administered IV at 0.05, 0.3, $1,3,10$ or $20 \mathrm{mg} / \mathrm{kg}$ or $\mathrm{SC}$ at $50 \mathrm{mg}$ at ratio of $2: 1$ or $3: 1$. Blood samples were obtained before and after dose administration up to week 16. Target modulation of BDCA2 and serum BDCA2 concentration were measured using flow cytometry, and an enzyme-linked immunosorbent assay (ELISA), respectively, and anti-drug antibodies (ADA) were measured using a bridging ELISA.

Results: 54 HV (38 BIIB059; 16 placebo) were assigned to cohort 1-7. BIIB059 demonstrated non-linear PK consistent with target mediated drug disposition. Bioavailability of SC administration was $\sim 50 \%$. Treatment with BIIB059 led to rapid and complete down-modulation of BDCA2 on the surface of pDCs at all dose levels. Time to reappearance of BDCA2 on pDC cell surface correlated with circulating levels of BIIB059, establishing an in vivo pharmacokinetic/pharmacodynamics (PK/PD) relationship. Single dose administration of BlIB059 was well tolerated across all dose levels. All AEs were mild to moderate in severity with no serious AEs. There were no clinically significant changes in laboratory assessments, physical examinations, or electrocardiogram (ECG) values. Anti-drug antibodies were detected at low levels in a small number of BIIB059-treated subjects. Responses were largely transient and demonstrated no impact on BIIB059 exposure or association with any safety event.

Conclusions: The first-in-human study in HV demonstrated acceptable safety/tolerability and PK profile in healthy subjects and supports further multipledose studies with BIIB059. BIIB059 treatment showed dose dependent target engagement and internalization of BDCA2 establishing a PK/PD correlation in vivo on circulating $\mathrm{pDC}$. These data support further evaluation of clinical efficacy and safety of BIIB059 as a potential novel therapy in SLE patients.

Acknowledgements: None

Disclosure of Interest: D. Martin Shareholder of: Biogen, Employee of: Biogen at the time this abstract was written, L. Stevenson Shareholder of: Biogen, Employee of: Biogen, P. Prasad Shareholder of: Biogen, Employee of: Biogen at the time this abstract was written, K. Smirnakis Shareholder of: Biogen, Employee of: Biogen, A. Kao Shareholder of: Biogen, Employee of: Biogen at the time this abstract was written, D. Rabah Shareholder of: Biogen, Employee of: Biogen, W. Wang Shareholder of: Biogen, Employee of: Biogen, C. Barbey Shareholder of: Biogen, Employee of: Biogen, C. Musselli Shareholder of: Biogen, Employee of: Biogen, N. Franchimont Shareholder of: Biogen, Employee of: Biogen

DOI: 10.1136/annrheumdis-2017-eular 6109 